\title{
Managing Eating Disorders on a General Pediatrics Unit: A Centralized Video Monitoring Pilot
}

\author{
Kristin A Shadman, MD*; Ryan J Coller, MD, MPH'; Windy Smith, MSN²; Michelle M Kelly, MD; Paula Cody, MD; \\ William Taft, MD'; Laura Bodine, RD²; Daniel J Sklansky, MD
}

${ }^{1}$ Department of Pediatrics, University of Wisconsin School of Medicine and Public Health, Madison, Wisconsin; ${ }^{2}$ American Family Children's Hospital, University of Wisconsin Hospital and Clinics, Madison, Wisconsin.

\begin{abstract}
Adolescents with severe eating disorders require hospitalization for medical stabilization. Supervision best practices for these patients are not established. This study sought to evaluate the cost and feasibility of centralized video monitoring (CVM) supervision on a general pediatric unit of an academic quaternary care center. This was a retrospective cohort study of nursing assistant (NA) versus CVM supervision for girls 12-18 years old admitted for medical stabilization of an eating disorder between September 2013 and March 2017. There were 37 consecutive admissions (NA = 23 and $\mathrm{CVM}=14$ ). NA median supervision cost was more expensive
\end{abstract}

than CVM $(\$ 4,104 /$ admission vs $\$ 1,166 /$ admission, $P<$ .001). Length of stay and days to weight gain were not statistically different. There were no occurances of family refusal of CVM, conversion from CVM to NA, technological failure, or unplanned discontinuation. Video monitoring was feasible and associated with lower supervision costs than one-to-one NA supervision. Larger samples in multiple centers are needed to confirm the safety, acceptability, and efficacy of CVM. Journal of Hospital Medicine 2019;14:357360. Published online first April 8, 2019. (C) 2019 Society of Hospital Medicine
H ospitalizations for nutritional rehabilitation of patients with restrictive eating disorders are increasing. ${ }^{1}$ Among primary mental health admissions at free-standing children's hospitals, eating disorders represent $5.5 \%$ of hospitalizations and are associated with the longest length of stay (LOS; mean 14.3 days) and costliest care (mean $\$ 46,130$ ). ${ }^{2}$ Admission is necessary to ensure initial weight restoration and monitoring for symptoms of refeeding syndrome, including electrolyte shifts and vital sign abnormalities. . $^{3-5}$

Supervision is generally considered an essential element of caring for hospitalized patients with eating disorders, who may experience difficulty adhering to nutritional treatment, perform excessive movement or exercise, or demonstrate purging or self-harming behaviors. Supervision is presumed to prevent counterproductive behaviors, facilitating weight gain and earlier discharge to psychiatric treatment. Best practices for patient supervision to address these challenges have not been established but often include meal time or continuous one-to-one supervision by nursing assistants (NAs) or other staff., 6 While meal supervision has been shown to decrease medical LOS, it is costly, reduces staff availability for the care of other patient care, and can be a barrier to caring for patients with eating disorders in many institutions. ${ }^{8}$

\footnotetext{
*Corresponding Author: Kristin A Shadman, MD; E-mail: kshadman@pediatrics.wisc.edu; Telephone: 608-265-8561.
}

Received: October 20, 2018; Revised: January 27, 2019;

Accepted: January 27, 2019

๑) 2019 Society of Hospital Medicine DOI 10.12788/jhm.3176
Although not previously used in patients with eating disorders, centralized video monitoring (CVM) may provide an additional mode of supervision. CVM is an emerging technology consisting of real-time video streaming, without video recording, enabling tracking of patient movement, redirection of behaviors, and communication with unit nurses when necessary. CVM has been used in multiple patient safety initiatives to reduce falls, address staffing shortages, reduce costs,, 10 supervise patients at risk for self-harm or elopement, and prevent controlled medication diversion. ${ }^{10,11}$

We sought to pilot a novel use of CVM to replace our institution's standard practice of continuous one-to-one nursing assistant (NA) supervision of patients admitted for medical stabilization of an eating disorder. Our objective was to evaluate the supervision cost and feasibility of CVM, using LOS and days to weight gain as balancing measures.

\section{METHODS}

\section{Setting and Participants}

This retrospective cohort study included patients 12-18 years old admitted to the pediatric hospital medicine service on a general unit of an academic quaternary care children's hospital for medical stabilization of an eating disorder between September 2013 and March 2017. Patients were identified using administrative data based on primary or secondary diagnosis of anorexia nervosa, eating disorder not other wise specified, or another specified eating disorder (ICD 93071 , 20759, or ICD 10 f5000, 5001, f5089, f509). ${ }^{12,13}$ This research study was considered exempt by the University of Wisconsin School of Medicine and Public Health's Institutional Review Board. 
TABLE 1. Characteristics of Patients Admitted for Eating Disorder Medical Stabilization

\begin{tabular}{lcc}
\hline & $\begin{array}{c}\text { Nursing Assistant } \\
\text { Supervision } \\
(\mathbf{n}=\mathbf{2 3}) \\
\%\end{array}$ & $\begin{array}{c}\text { Central Video Monitoring } \\
\text { Supervision } \\
(\mathbf{n}=14) \\
\%\end{array}$ \\
\hline Gender, female & 100 & 100 \\
\hline Age in years, mean & 14.8 & 15.0 \\
\hline Race, ethnicity & & \\
White, non-Hispanic & 92 & 79 \\
White, Hispanic & 4 & 7 \\
Asian & 0 & 7 \\
Black, non-Hispanic & 0 & 0 \\
Black, Hispanic & 0 & 0 \\
Other/Declined & 4 & 7 \\
\hline Insurance & & \\
Private & 80 & 72 \\
Public & 20 & 14 \\
\hline Suicidality & 9 & 28 \\
\hline
\end{tabular}

\section{Supervision Interventions}

A standard medical stabilization protocol was used for patients admitted with an eating disorder throughout the study period (Appendix). All patients received continuous one-to-one NA supervision until they reached the target calorie intake and demonstrated the ability to follow the nutritional meal protocol. Beginning July 2015, patients received continuous CVM supervision unless they expressed suicidal ideation (SI), which triggered one-to-one NA supervision until they no longer endorsed suicidality.

\section{Centralized Video Monitoring Implementation}

Institutional CVM technology was AvaSys TeleSitter Solution (AvaSure, Inc). Our institution purchased CVM devices for use in adult settings, and one was assigned for pediatric CVM. Mobile CVM video carts were deployed to patient rooms and generated live video streams, without recorded capture, which were supervised by CVM technicians. These technicians were NAs hired and trained specifically for this role; worked four-, eight-, and 12-hour shifts; and observed up to eight camera feeds on a single monitor in a centralized room. Patients and family members could refuse CVM, which would trigger one-to-one NA supervision. Patients were not observed by CVM while in the restroom; staff were notified by either the patient or technician, and one-to-one supervision was provided. CVM had two-way audio communication, which allowed technicians to redirect patients verbally. Technicians could contact nursing staff directly by phone when additional intervention was needed.

\section{Supervision Costs}

NA supervision costs were estimated at $\$ 19 /$ hour, based upon institutional human resources average NA salaries at that time. No additional mealtime supervision was included, as in-person supervision was already occurring.
CVM supervision costs were defined as the sum of the device cost plus CVM technician costs and two hours of one-toone NA mealtime supervision per day. The CVM device cost was estimated at $\$ 2.10 /$ hour, assuming a 10-year machine life expectancy (single unit cost $\$ 82,893$ in 2015, 3,944 hours of use in fiscal year of 2018). CVM technician costs were $\$ 19 /$ hour, based upon institutional human resources average CVM technician salaries at that time. Because technicians monitored an average of six patients simultaneously during this study, onesixth of a CVM technician's salary (ie, \$3.17/hour) was used for each hour of CVM monitoring. Patients with mixed (NA and CVM) supervision were analyzed with those having CVM supervision. These patients' costs were the sum of their NA supervision costs plus their CVM supervision costs.

\section{Data Collection}

Descriptive variables including age, gender, race/ethnicity, insurance, and LOS were collected from administrative data. The duration and type of supervision for all patients were collected from daily staffing logs. The eating disorder protocol standardized the process of obtaining daily weights (Appendix). Days to weight gain following admission were defined as the total number of days from admission to the first day of weight gain that was followed by another day of weight gain or maintaining the same weight. CVM acceptability and feasibility were assessed by family refusal of CVM, conversion from CVM to NA, technological failure, complaints, and unplanned discontinuation, which were prospectively documented by the unit nurse manager.

\section{Data Analysis}

Patient and hospitalization characteristics were summarized. A sample size of at least 14 in each group was estimated as necessary to detect a $50 \%$ reduction in supervision cost between the groups using alpha $=0.05$, a power of $80 \%$, a mean cost of $\$ 4,400$ in the NA group, and a standard deviation of $\$ 1,600$. Wilcoxon rank-sum tests were used to assess differences in median supervision cost between NA and CVM use. Differences in mean LOS and days to weight gain between NA and CVM use were assessed with $t$-tests because these data were normally distributed.

\section{RESULTS}

\section{Patient Characteristics and Supervision Costs}

The study included 37 consecutive admissions (NA $=23$ and $C V M=14)$ with 35 unique patients. Patients were female, primarily non-Hispanic White, and privately insured (Table 1). Median supervision cost for the NA was statistically significantly more expensive at $\$ 4,104 /$ admission versus $\$ 1,166 /$ admission for CVM $(P<.001$, Table 2).

Balancing Measures, Acceptability, and Feasibility Mean LOS was 11.7 days for NA and 9.8 days for CVM ( $P=$ .27; Table 2). The mean number of days to weight gain was 3.1 and 3.6 days, respectively $(P=.28)$. No patients converted from CVM to NA supervision. One patient with SI converted 


\begin{tabular}{|c|c|c|c|c|}
\hline & $\begin{array}{l}\text { Nursing Assistant Supervision } \\
\qquad(\mathrm{n}=23)\end{array}$ & $\begin{array}{l}\text { Centralized Video } \\
\text { Monitoring Supervision } \\
\qquad(\mathrm{n}=14)\end{array}$ & $P$ Value & $\begin{array}{l}\text { Differences } \\
(95 \% \mathrm{CI})\end{array}$ \\
\hline Supervision cost, median, \$ (USD) & 4,104 & 1,166 & $<.001$ & $\begin{array}{c}2,938 \\
(1,998-3,942)\end{array}$ \\
\hline Cost attributed to nursing assistant supervision, (\%) & 100 & 39.2 & $<.001$ & NA \\
\hline Monitoring time, median, hours & 216 & 182 & .29 & $\begin{array}{c}34 \\
(-31.9-103)\end{array}$ \\
\hline Length of stay, mean, days & 11.7 & 9.8 & 0.27 & $\begin{array}{c}1.9 \\
(-1.5-0.4)\end{array}$ \\
\hline Days to weight gain, mean & 3.1 & 3.6 & 0.28 & $\begin{array}{c}-0.5 \\
(-1.4-5.3)\end{array}$ \\
\hline
\end{tabular}

to CVM after SI resolved and two patients required ongoing NA supervision due to continued SI. There were no reported refusals, technology failures, or unplanned discontinuations of CVM. One patient/family reported excessive CVM redirection of behavior.

\section{DISCUSSION}

This is the first description of CVM use in adolescent patients or patients with eating disorders. Our results suggest that CVM appears feasible and less costly in this population than one-toone NA supervision, without statistically significant differences in LOS or time to weight gain. Patients with CVM with any NA supervision (except mealtime alone) were analyzed in the CVM group; therefore, this study may underestimate cost savings from CVM supervision. This innovative use of CVM may represent an opportunity for hospitals to repurpose monitoring technology for more efficient supervision of patients with eating disorders.

This pediatric pilot study adds to the growing body of literature in adult patients suggesting CVM supervision may be a feasible inpatient cost-reduction strategy. 9,10 One single-center study demonstrated that the use of CVM with adult inpatients led to fewer unsafe behaviors, eg, patient removal of intravenous catheters and oxygen therapy. Personnel savings exceeded the original investment cost of the monitor within one fiscal quarter. ${ }^{9}$ Results of another study suggest that CVM use with hospitalized adults who required supervision to prevent falls was associated with improved patient and family satisfaction. ${ }^{14}$ In the absence of a gold standard for supervision of patients hospitalized with eating disorders, CVM technology is a tool that may balance cost, care quality, and patient experience. Given the upfront investment in CVM units, this technology may be most appropriate for institutions already using CVM for other inpatient indications.

Although our institutional cost of CVM use was similar to that reported by other institutions, ${ }^{11,15}$ the single-center design of this pilot study limits the generalizability of our findings. Unadjusted results of this observational study may be confounded by indication bias. As this was a pilot study, it was powered to detect a clinically significant difference in cost between NA and CVM supervision. While statistically significant differences were not seen in LOS or weight gain, this pilot study was not powered to detect potential differences or to adjust for all potential confounders (eg, other mental health conditions or comorbidities, eating disorder type, previous hospitalizations). Future studies should include these considerations in estimating sample sizes. The ability to conduct a robust cost-effectiveness analysis was also limited by cost data availability and reliance on staffing assumptions to calculate supervision costs. However, these findings will be important for valid effect size estimates for future interventional studies that rigorously evaluate CVM effectiveness and safety. Patients and families were not formally surveyed about their experiences with CVM, and the patient and family experience is another important outcome to consider in future studies.

\section{CONCLUSION}

The results of this pilot study suggest that supervision costs for patients admitted for medical stabilization of eating disorders were statistically significantly lower with CVM when compared with one-to-one NA supervision, without a change in hospitalization LOS or time to weight gain. These findings are particularly important as hospitals seek opportunities to reduce costs while providing safe and effective care. Future efforts should focus on evaluating clinical outcomes and patient experiences with this technology and strategies to maximize efficiency to offset the initial device cost.

Disclosures: The authors have no financial relationships relevant to this article to disclose. The authors have no conflicts of interest relevant to this article to disclose.

\section{References}

1. Zhao $Y$, Encinosa W. An update on hospitalizations for eating disorders, 1999 to 2009: statistical brief \#120. In: Healthcare Cost and Utilization Project (HCUP) Statistical Briefs. Rockville, MD: Agency for Healthcare Research and Quality (US); 2006.

2. Bardach NS, Coker TR, Zima BT, et al. Common and costly hospitalizations 
for pediatric mental health disorders. Pediatrics. 2014;133(4):602-609. doi 10.1542/peds.2013-3165.

3. Society for Adolescent H, Medicine, Golden NH, et al. Position Paper of the Society for Adolescent Health and Medicine: medical management of restrictive eating disorders in adolescents and young adults. J Adolesc Health. 2015;56(1):121-125. doi: 10.1016/j.jadohealth.2014.10.259.

4. Katzman DK. Medical complications in adolescents with anorexia nervosa: a review of the literature. Int J Eat Disord. 2005;37(S1):S52-S59; discussion S87-S59. doi: 10.1002/eat.20118.

5. Strandjord SE, Sieke EH, Richmond M, Khadilkar A, Rome ES. Medical stabilization of adolescents with nutritional insufficiency: a clinical care path. Eat Weight Disord. 2016;21(3):403-410. doi: 10.1007/s40519-015-0245-5.

6. Kells M, Davidson K, Hitchko L, O'Neil K, Schubert-Bob P, McCabe M. Examining supervised meals in patients with restrictive eating disorders. Appl Nurs Res. 2013;26(2):76-79. doi: 10.1016/j.apnr.2012.06.003.

7. Leclerc A, Turrini T, Sherwood K, Katzman DK. Evaluation of a nutrition rehabilitation protocol in hospitalized adolescents with restrictive eating disorders. J Adolesc Health. 2013;53(5):585-589. doi: 10.1016/j.jadohealth.2013.06.001.

8. Kells M, Schubert-Bob P, Nagle K, et al. Meal supervision during medical hospitalization for eating disorders. Clin Nurs Res. 2017;26(4):525-537. doi: 10.1177/1054773816637598.

9. Jeffers $S$, Searcey P, Boyle K, et al. Centralized video monitoring for patient safety: a Denver Health Lean journey. Nurs Econ. 2013;31(6):298-306.

10. Sand-Jecklin K, Johnson JR, Tylka S. Protecting patient safety: can video monitoring prevent falls in high-risk patient populations? J Nurs Care Qual. 2016;31(2):131-138. doi: 10.1097/NCQ.0000000000000163.

11. Burtson PL, Vento L. Sitter reduction through mobile video monitoring: a nurse-driven sitter protocol and administrative oversight. J Nurs Adm. 2015;45(7-8):363-369. doi: 10.1097/NNA.0000000000000216.

12. Prevention CfDCa. ICD-9-CM Guidelines, 9th ed. https://www.cdc.gov/nchs/ data/icd/icd9cm_guidelines_2011.pdf. Accessed April 11, 2018.

13. Prevention CfDca. IDC-9-CM Code Conversion Table. https://www.cdc.gov/ nchs/data/icd/icd-9-cm_fy14_cnvtbl_final.pdf. Accessed April 11, 2018.

14. Cournan M, Fusco-Gessick B, Wright L. Improving patient safety through video monitoring. Rehabil Nurs. 2016. doi: 10.1002/rnj.308.

15. Rochefort CM, Ward L, Ritchie JA, Girard N, Tamblyn RM. Patient and nurse staffing characteristics associated with high sitter use costs. J Adv Nurs. 2012;68(8):1758-1767. doi: 10.1111/j.1365-2648.2011.05864.x. 